\title{
Analysis of the risk factors for influenza A (H1N1) pneumonia
}

\author{
QING ZHANG $^{1 *}$, ZHI-WEI ZHAO $^{1 *}$, ZHI-LI XING $^{2}$, BO ZHANG $^{1}$, HONG-FEI ZHENG ${ }^{1}$ and LI-XIN SUN ${ }^{3}$ \\ Departments of ${ }^{1}$ Respiratory Disease and ${ }^{2}$ Emergency, and ${ }^{3}$ Central Laboratory, The Affiliated \\ Hospital of Chengde Medical College, Chengde, Hebei 067000, P.R. China
}

Received April 4, 2013; Accepted May 16, 2013

DOI: $10.3892 /$ br.2013.128

\begin{abstract}
Pneumonia is the most common complication of influenza A (H1N1). However, there has been no identification of any single initial symptom as an independent risk factor. In 2009, 206 patients were diagnosed with H1N1 in the Chengde area, China and they were assembled in the Chengde Hospital for Infectious Diseases. The diagnosis and treatment were performed in accordance with the Protocol for Diagnosis and Treatment of Influenza A (H1N1) (2009, third edition), issued by the General Office of the Ministry of Health, with detailed records by the medical staff assigned by the Bureau of Health of the Chengde government. All the patients had viral nucleic acid-positive throat swabs detected with quantitative reverse transcription polymerase chain reaction (qPCR). Based on the final imaging findings, patients were divided into the pneumonia and non-pneumonia groups for this case control study. The univariate analysis demonstrated that the ratios of patients aged $0-5$ and $\geq 45$ years, with underlying diseases, with initial symptoms including cough, expectoration and dyspnea and with onset-to-treatment interval of $>48 \mathrm{~h}$ were higher in the pneumonia compared to the non-pneumonia group $(\mathrm{P}<0.001, \mathrm{P}<0.001, \mathrm{P}=0.018, \mathrm{P}<0.001, \mathrm{P}<0.001$ and $\mathrm{P}<0.001$, respectively). The multivariate logistic regression analysis demonstrated that age 0-5 years, presence of underlying diseases, expectoration as the initial symptom and onset-to-treatment interval of $>48 \mathrm{~h}$ were the independent risk factors for pneumonia with relative odds ratios (OR) and 95\% confidence intervals (95\% CIs) of 6.120 and 1.779-21.088, 11.188 and 2.021-61.935, 5.263 and 2.042-13.562, and 22.873 and 6.110-85.631, respectively $(\mathrm{P}<0.01)$. Therefore, it is recommended that patients with $\mathrm{H} 1 \mathrm{~N} 1$ presenting with expectoration as the initial symptom be treated with caution during influenza pandemics.
\end{abstract}

Correspondence to: Professor Li-Xin Sun, Central Laboratory, The Affiliated Hospital of Chengde Medical College, Nanyingzi Street 36, Chengde, Hebei 067000, P.R. China

E-mail: cyfyzys@163.com

*Contributed equally

Key words: influenza A (H1N1), pneumonia, risk factor, case control study

\section{Introduction}

In April 2009, there was an outbreak of novel influenza A (H1N1) in the United States of America and Mexico. On June 11, 2009, the World Health Organization declared a global pandemic (1). The most common complication of this outbreak was pneumonia, which became the primary cause of critical illness and mortality (2). By August 1, 2010, at least 18,449 patients had succumbed to the infection worldwide (3). In the face of an $\mathrm{H} 1 \mathrm{~N} 1$ pandemic, there was a surge of a large number of patients within a short period of time, with some suffering from pneumonia, which resulted in death in severe cases. However, the incidence and mortality rate of pneumonia may be reduced if the risk factors for susceptibility to pneumonia are identified and high-risk patients are diagnosed and treated early in the course of the disease. Therefore, in this study, the patients with H1N1 who were assembled in the Chengde Hospital for Infectious Diseases for free treatment were assessed to identify the risk factors for susceptibility to pneumonia and provide a basis for the prevention and treatment of $\mathrm{H} 1 \mathrm{~N} 1$ pandemics in the future.

\section{Subjects and methods}

Subjects. Patients with suspected influenza in the Chengde area were isolated and screened centrally in the Chengde Hospital for Infectious Diseases between September and December, 2009. A total of 206 patients with H1N1 infection, whose throat swabs tested positive for viral H1N1 nucleic acid using quantitative reverse transcription polymerase chain reaction (qPCR), were hospitalized for isolation and treatment. The patients with excluded H1N1 infection were treated at the local community or other hospitals. All the patients were diagnosed according to the criteria described in the Protocol for Diagnosis and Treatment of Influenza A (H1N1) (2009, third edition) (4), issued by the General Office of the Ministry of Health. These patients from 8 counties and 3 districts in Chengde were diagnosed and treated by healthcare specialists who were centrally deployed by the Chengde Bureau of Health from the Departments of Respiratory Medicine, Emergency Medicine and Pediatrics. The qPCR test was conducted by the Center for Disease Control and Prevention of Chengde. The initial throat swab samples were delivered to the Center for Disease Control and Prevention of Hebei, China, for verification.

Methods. Based on the final imaging findings, the patients were divided into the pneumonia $(n=51)$ and non-pneumonia 
$(\mathrm{n}=155)$ groups. Gender, age, body mass index (BMI), underlying diseases, initial symptoms at disease onset and onset-to-treatment interval (the treatment time in this study was defined as the time when antiviral drugs were administered) in the 2 groups were investigated in this study. Furthermore, the patients were stratified by age into $0-5,6-44$, $45-59$ and $\geq 60$ years groups for analysis. Since only 5 patients aged $\geq 60$ years developed pneumonia in this study, the 45-59 and $\geq 60$ years groups were combined into the $\geq 45$ years group.

Statistical analysis. SPSS17.0 software was used for statistical analysis of all data. Measurement data were presented as median (interquartile range) $[\mathrm{M}(\mathrm{IQR})]$, whereas numeration data were presented as the constituent ratio (\%). A univariate analysis was performed with the Mann-Whitney U-test for measurement data and the $\chi^{2}$ or Fisher's exact probability test for comparison of the constituent ratios. A multivariate analysis was performed using the forward multivariate logistic regression analysis. $\mathrm{P}<0.05$ was considered to indicate a statistically significant difference.

\section{Results}

General data. This study included a total of 206 patients, 144 of whom were men $(69.9 \%)$ and 62 women (30.1\%), aged 7 months- 80 years, with a median age of 18.5 years (range, 11-22 years). Seventeen patients had underlying diseases and 11 were aged $\geq 45$ years; chronic lung disease was the most common underlying illness, encountered in 9 patients, 3 of whom had a concomitant complication of chronic cardiovascular disease. The occupational distribution of these patients was as follows: 142 registered students (69 from the same university), 24 preschool children, 15 unemployed and retired urban residents, 14 farmers, 6 workers and 5 in other professions. Fifty-three cases were severe, including 25 critically ill patients, 9 who received mechanical ventilation, 5 with acute respiratory distress syndrome and 3 mortality cases. The remaining patients were cured and discharged.

Univariate analysis between pneumonia and non-pneumonia groups. The difference in the ratio of patients of male gender and $\mathrm{BMI} \geq 30 \mathrm{~kg} / \mathrm{m}^{2}$ was of no statistical significance $(\mathrm{P}=0.318)$. The median age was 8 years higher in the pneumonia compared to the non-pneumonia group $(\mathrm{P}=0.001)$. The ratios of patients aged $0-5$ and $\geq 45$ years were statistically higher in the pneumonia compared to the non-pneumonia group (both P-values $<0.001)$. The ratio of patients with underlying or chronic lung diseases was statistically higher in the pneumonia compared to the non-pneumonia group $(\mathrm{P}<0.001)$. The ratio of patients with initial symptoms such as cough, expectoration and dyspnea was higher in the pneumonia compared to the non-pneumonia group, with statistically significant differences (all P-values <0.05). The ratio of patients with initial symptoms such as fever and headache was lower in the pneumonia compared to the non-pneumonia group, with differences of statistical significance. The median onset-to-treatment time was 4 days longer in the pneumonia compared to the non-pneumonia group, with a statistically significant difference $(\mathrm{P}<0.001)$. The ratio of patients who were treated $>48 \mathrm{~h}$ after the onset was higher in the pneumonia compared to the non-pneumonia group, with a statistically significant difference $(\mathrm{P}<0.001$; Table I).

Multivariate analysis. Forward multivariate logistic regression analysis was performed using progression into pneumonia as the dependent variable and factors of statistical significance in the univariate analysis as the independent variables. The results identified 0-5 years of age, presence of underlying diseases, expectoration as the initial symptom and $>48 \mathrm{~h}$ from disease onset to treatment as independent risk factors for susceptibility to pneumonia $(\mathrm{P}<0.05)$ (Table II).

\section{Discussion}

The genome of influenza A (H1N1) responsible for this outbreak is a novel mutant resulting from the reassortment of gene fragments from 3 sources: avian, swine and human flu viruses (5). Due to the absence of prior exposure to this virus, the body did not have antibodies against it and recent vaccination against seasonal influenza did not produce cross-reaction antibodies against this virus (6); therefore, the population was susceptible to H1N1. However, not all the infected individuals developed pneumonia and the patients with pneumonia exhibited certain risk factors. As demonstrated by this study, children aged 0-5 years, middle-aged and elderly individuals ( $\geq 45$ years), the presence of chronic underlying diseases, initial symptoms such as cough, expectoration and dyspnea and $>48 \mathrm{~h}$ from disease onset to treatment were the risk factors determining susceptibility to pneumonia in patients with H1N1. By contrast, gender, adolescence, BMI $\geq 30 \mathrm{~kg} / \mathrm{m}^{2}$ and initial symptoms such as fever, sore throat, headache, vomiting and diarrhea were not identified as risk factors for susceptibility to pneumonia in patients with H1N1. Although previous studies suggested $\mathrm{BMI} \geq 30 \mathrm{~kg} / \mathrm{m}^{2}$ as a risk factor for progression into critical conditions in patients with H1N1 $(7,8)$, in this study, the difference in the ratio of patients with BMI $\geq 30 \mathrm{~kg} / \mathrm{m}^{2}$ between the pneumonia and the non-pneumonia group was of no statistical significance, possibly due to the different patient population. A further multivariate logistic regression analysis identified age of 0-5 years, presence of underlying diseases, expectoration as the initial symptom and $>48 \mathrm{~h}$ from disease onset to treatment as the independent risk factors for susceptibility to pneumonia in patients with H1N1.

This study identified children aged 0-5 years as an age group susceptible to pneumonia and the risk to develop pneumonia was 6.12-fold higher in patients aged 0-5 years compared to those aged 6-44 years, with a $95 \% \mathrm{CI}$ of $1.776-21.088$. The number of children aged 0-5 years and middle-aged and elderly patients aged $\geq 45$ years was relatively small, constituting $17.5 \%$ of the total patient sample and elderly patients aged $\geq 60$ years represented only $2.4 \%$ of all patients. This may be attributed to the limited social activities of these 2 age groups and, consequently, the limited opportunities for contagious infection, as well as to the presence of antiviral antibody against H1N1 in the blood of some elderly patients aged $>60$ years (6), leading to a lower incidence rate compared to adolescents. However, the ratio of patients with pneumonia was relatively higher among those aged 0-5 and $\geq 45$ years in this study, thus the ratios of patients aged $0-5$ and $\geq 45$ years were higher in the pneumonia group compared to the non-pneumonia group. A possible 
Table I. Comparison of clinical characteristics between the pneumonia group and the non-pneumonia groups.

\begin{tabular}{|c|c|c|c|}
\hline Characteristics & $\begin{array}{l}\text { Pneumonia group } \\
\quad \text { (51 patients) }\end{array}$ & $\begin{array}{l}\text { Non-pneumonia group } \\
\text { (155 patients) }\end{array}$ & P-value \\
\hline \multicolumn{4}{|l|}{ Gender, n (\%) } \\
\hline Men & $31(60.8)$ & $106(68.4)$ & 0.318 \\
\hline Women & $20(39.2)$ & $49(31.6)$ & \\
\hline Median age $[\mathrm{M}(\mathrm{IQR})$ years], n (\%) & $26(6-45)$ & $18(12-21)$ & 0.001 \\
\hline $0-5$ & $12(23.5)$ & $6(3.9)$ & $<0.001$ \\
\hline $6-44$ & $25(49.0)$ & $147(94.8)$ & $<0.001$ \\
\hline$\geq 45$ & $14(27.5)$ & $2(1.3)$ & $<0.001$ \\
\hline $\mathrm{BMI} \geq 30 \mathrm{~kg} / \mathrm{m}^{2}, \mathrm{n}(\%)$ & $7(13.7)$ & $10(6.5)$ & 0.139 \\
\hline Underlying diseases, n (\%) & $13(25.5)$ & $4(2.6)$ & $<0.001$ \\
\hline Chronic lung diseases, n (\%) & $8(15.7)$ & $1(0.6)$ & $<0.001$ \\
\hline \multicolumn{4}{|l|}{ Initial symptoms, n (\%) } \\
\hline Fever & $33(64.7)$ & $142(91.6)$ & $<0.001$ \\
\hline Cough & $43(84.3)$ & $103(67.3)$ & 0.018 \\
\hline Expectoration & $32(62.7)$ & $41(26.5)$ & $<0.001$ \\
\hline Sore throat & $6(11.8)$ & $31(20.0)$ & 0.184 \\
\hline Dyspnea & $10(19.6)$ & $1(0.6)$ & $<0.001$ \\
\hline Headache & $2(3.9)$ & $29(18.7)$ & 0.010 \\
\hline Vomiting & $1(2.0)$ & $10(6.5)$ & 0.299 \\
\hline Diarrhea & 0 & $2(1.3)$ & 1.0 \\
\hline Median onset-to-treatment time $[\mathrm{M}(\mathrm{IQR}) \mathrm{d}], \mathrm{n}(\%)$ & $6(4-7)$ & $2(1-3)$ & $<0.001$ \\
\hline$\leq 48 \mathrm{~h}$ & $3(5.9)$ & $110(71.0)$ & $<0.001$ \\
\hline$>48 \mathrm{~h}$ & $48(94.1)$ & $45(29.0)$ & \\
\hline
\end{tabular}

IQR, interquartile range; BMI, body mass index; d, days.

Table II. Results of the multivariate logistic regression analysis of the risk factors for influenza A (H1N1) pneumonia.

\begin{tabular}{|c|c|c|c|c|}
\hline Variable & Wald value & P-value & OR-value & $95 \% \mathrm{CI}$ \\
\hline Age (years) & 10.496 & 0.005 & & \\
\hline $0-5$ & 8.263 & 0.004 & 6.120 & $1.776-21.088$ \\
\hline$\geq 45$ & 3.419 & 0.064 & 5.633 & $0.902-35.195$ \\
\hline Underlying diseases & 7.650 & 0.006 & 11.188 & $2.021-13.562$ \\
\hline Expectoration as initial symptom & 11.822 & 0.001 & 5.263 & $2.042-13.562$ \\
\hline Onset-to-treatment time $>48 \mathrm{~h}$ & 21.596 & $<0.001$ & 22.873 & $6.110-85.631$ \\
\hline
\end{tabular}

OR, odds ratio; CI, confidence interval.

explanation is the relatively poor immunity of children and middle-aged and elderly individuals. This was consistent with the observation of Louie et al (9), who reported that the population exhibiting severe disease conditions in this influenza A pandemic was dominated by infants, young children and patients aged $\geq 50$ years (9). It was demonstrated by combining the results of the multivariate analysis that the age of $0-5$ years was an independent risk factor for susceptibility to pneumonia, which was consistent with the WHO report on children aged $<5$ years being prone to progression into critical illness (2).
This study demonstrated that the risk of developing pneumonia was 11.18 -fold higher in patients with compared to those without underlying diseases, with a 95\% CI of 2.021-61.935. The ratio of chronic lung diseases was higher in patients with pneumonia and 3 patients exhibited a concomitant complication of chronic cardiovascular disease, which was consistent with previous studies, reporting that chronic lung and cardiovascular diseases in patients with H1N1 were risk factors for progression into critical conditions $(10,11)$. The initial symptoms manifested by pneumonia patients in this study were 
dominated by cough, expectoration and dyspnea. Chronic underlying diseases, particularly lung diseases, decrease the innate defense mechanism of the respiratory system of the patients, allowing the $\mathrm{H} 1 \mathrm{~N} 1$ virus to enter the lower respiratory tract, aggravate the underlying disease and cause pneumonia.

Although a comparison of the initial symptoms between pneumonia and non-pneumonia patients has rarely been seen in previous studies, the initial symptoms at disease onset may better reflect patient characteristics in the pneumonia group prior to the onset of pneumonia and, to a certain extent, reflect the site of viral infection in the early stages of the disease. In this study, the initial symptoms of pneumonia patients were mainly cough, expectoration and dyspnea, as the virus infected the lower respiratory tract of pneumonia patients at the early stage of the disease, leading to hyperemia, edema and increased mucus secretion of the respiratory mucosa. By contrast, the initial symptoms of non-pneumonia patients were mainly fever and headache, which was due to the virus not affecting the lower respiratory tract, with the manifestation of only mild local respiratory symptoms. This study demonstrated that the risk for developing pneumonia was 5.3-fold higher in patients with expectoration as the initial symptom compared to those without expectoration, with a $95 \% \mathrm{CI}$ of 2.024-13.562. Since expectoration is one of the main symptoms of lower respiratory tract infection, when it appeared as an initial symptom it suggested that the virus had infected the lower respiratory tract at the early stage of the disease, rendering the patients prone to the development of pneumonia, as they had not received any antiviral drugs and the specific immune system had not been activated.

Previous studies used the interval of $>48 \mathrm{~h}$ from symptom manifestation to initiation of oseltamivir as a risk factor for susceptibility to progression into critical illness $(12,13)$. As shown in a multivariate analysis of risk factors for ICU hospitalization and death, involving 272 inpatients in the United States, a time of $>48 \mathrm{~h}$ from disease onset to initiation of oseltamivir was the only risk factor of significance (12). This study has demonstrated that the risk for developing pneumonia was 22.9-fold higher if onset-to-treatment time was $>48 \mathrm{~h}$ compared to $\leq 48 \mathrm{~h}$, with a 95\% CI of 6.110-85.631. The median onset-to-treatment time was 4 days longer in pneumonia compared to non-pneumonia patients. A small percentage of patients (5.9\%) were treated within $48 \mathrm{~h}$ and the onset-to-treatment time exceeded $48 \mathrm{~h}$ in $94.1 \%$ pneumonia patients. Considering that treatment time and administration of antiviral drugs were consistent in this study, it is possible that the antiviral drugs may have in fact prevented disease progression.

In summary, as shown by the analysis of data obtained from patients with a definitive diagnosis of H1N1 infection in the Chengde area, age 0-5 years, the presence of underlying diseases, expectoration as the initial symptom and $>48 \mathrm{~h}$ from disease onset to treatment were the independent risk factors for susceptibility to pneumonia in patients with influenza A. To the best of our knowledge, of the above-mentioned risk factors, the identification of expectoration as the initial symptom as an independent risk factor for susceptibility to pneumonia in patients with H1N1 has not been reported in the past.

Since the Chengde city government assembled all the patients from the Chengde area with a definitive H1N1 diagnosis in Chengde Hospital for Infectious Diseases for free treatment, all these patients were diagnosed and treated by members of the same expert panel and the same group of healthcare specialists, with the support and cooperation of the health administrations. As a result, biases were largely avoided to reflect the local situation more objectively, which was rarely the case in previous studies, despite the unavoidable limitations of this single-center study in the Chengde area. H1N1 infection as a risk factor for pneumonia may be further verified by future multicenter studies in order to reduce the incidence of pneumonia in patients with H1N1.

\section{References}

1. No authors listed: New influenza A (H1N1) virus: global epidemiological situation, June 2009. Wkly Epidemiol Rec 84: 249-257, 2009

2. Writing Committee of the WHO Consultation on Clinical Aspects of Pandemic (H1N1) 2009 Influenza, Bautista E, Chotpitayasunondh T, Gao Z, Harper SA, Shaw M, Uyeki TM, Zaki SR, Hayden FG, Hui DS, Kettner JD, Kumar A, Lim M, Shindo N, Penn C and Nicholson KG: Clinical aspects of pandemic 2009 influenza A (H1N1) virus infection. N Engl J Med 362: 1708-1719, 2010.

3. World Health Organization: Pandemic (H1N1) 2009-update 112 [EB/OL]. http://www.who.int/csr/don/2010_08_06/en/. Accessed October 13, 2009

4. General Office of the Ministry of Health, Program of diagnosis and treatment of influenza A H1N1 (Version III, 2009) [EB/OL]. http:// news.xinhuanet.com/health/2009-11/11/content_12431885_3.htm. Accessed October 13, 2009 (In Chinese).

5. Trifonov V, Khiabanian $\mathrm{H}$ and Rabadan R: Geographic dependence, surveillance, and origins of the 2009 influenza A (H1N1) virus. N Engl J Med 361: 115-119, 2009.

6. Hancock K, Veguilla V, Lu X, Zhong W, Butler EN, et al: Cross-reactive antibody responses to the 2009 pandemic H1N1 influenza virus. N Engl J Med 361: 1945-1952, 2009.

7. ANZIC Influenza Investigators, Webb SA, Pettilä V, Seppelt I, et al: Critical care services and $2009 \mathrm{H} 1 \mathrm{N1}$ influenza in Australia and New Zealand. N Engl J Med 361: 1925-1934, 2009.

8. Kumar A, Zarychanski R, Pinto R, et al: Critically ill patients with 2009 influenza A (H1N1) infection in Canada. JAMA 302: 1872-1879, 2009

9. Louie JK, Aeosta M, Willter K, et al: Factors associated with death or hospitalization due to pandemic 2009 influenza A (H1N1) infection in Califomia. JAMA 302: 1896-1902, 2009.

10. Van Kerkhove MD, Vandemaele KA, Shinde V, Jaramillo-Gutierrez G, Koukounari A, et al: Risk factors for severe outcomes following 2009 influenza A (H1N1) infection: a global pooled analysis. PLoS Med 8: e1001053, 2011.

11. Jain S, Kamimoto L, Bramley AM, Schmitz AM, Benoit SR, et al: Hospitalized patients with $2009 \mathrm{H} 1 \mathrm{~N} 1$ influenza in the United States, April-June 2009. N Engl J Med 361: 1935-1944, 2009.

12. Echevarría Zuno S, Mejía-Aranguré JM, Mar-Obeso AJ, et al: Infection and death from influenza A H1N1 virus in Mexico: a retrospective analysis. Lancet 374: 2072-2079, 2009.

13. Higuera Iglesias AL, Kudo K, Manabe T, Corcho Berdugo AE, Corrales Baeza AC, et al: Reducing occurrence and severity of pneumonia due to pandemic H1N1 2009 by early oseltamivir administration: a retrospective study in Mexico. PLoS One 6: e21838, 2011. 